\title{
ERRATA
}

\section{Equatorial electrojet in the East Brazil anomaly region}

R. G. Rastogi and K. Yumoto

Earth Planets Space, Vol. 59, pp. 103-106, 2007

The magnetic field component $[\mathrm{X}]$ used in the paper is the Horizontal component of the geomagneic field towards magnetic North generally expressed as $[\mathrm{H}]$. 